\title{
Regioselectivity of the Oxygen Addition- Induced Dechlorination of PCBs and DDT Metabolites in Electron Capture Mass Spectrometry*
}

\author{
F. L. Lépine and S. Milot \\ Institut Armand-Frappier, Laval, Québec, Canada \\ O. A. Mamer \\ Biomedical Mass Spectrometry Unit, McGill University, Montréal, Québec, Canada
}

\begin{abstract}
The electron capture mass spectra of $28{ }^{35} \mathrm{Cl}$-labeled polychlorinated biphenyls (PCBs) and $4{ }^{37} \mathrm{Cl}$-labeled 1,1,1-trichloro-2,2-bis ( $p$-chlorophenyl)ethane (DDT) metabolites were obtained by using a $20 \%$ oxygen in methane mixture as the reagent gas. The degree of regioselectivity of the PCB oxygen addition-induced dechlorination reaction was determined by measurement of the residual amount of label in the M-19 ions produced by addition of $\mathrm{O}_{2}$ and subsequent loss of $\mathrm{OCl}$ from the molecule. Chlorine was lost in a random manner from the PCBs, contrary to the dechlorination reaction observed when methane alone was used. For the DDT metabolites, many dechlorination reactions were observed in addition to the one that generated the M-19 ions. Loss of $\mathrm{Cl}$, loss of $\mathrm{Cl}_{2}$, and addition of $\mathrm{O}_{2}$ with the loss of one or two $\mathrm{HCl}$ molecules also were seen. These various dechlorination reactions involved only the aliphatic chlorines. Addition of $\mathrm{O}_{2}$ followed by loss of $\mathrm{Cl}$ at the beta position of 2,2-bis $\left(4-{ }^{37} \mathrm{Cl}\right.$-chlorophenyl)-1-chloroethylene and 2,2-bis $\left(4-{ }^{37} \mathrm{Cl}\right.$-chlorophenyl)-1,1-dichloroethylene may be due to the ability of the diphenyl methane moiety to stabilize the intermediates. Formation of an ion that corresponds to 4,4'-dichlorobenzophenone also was observed for three of these labeled DDT metabolites. (J Am Soc Mass Spectrom 1996, 7, 66-72)
\end{abstract}

$\mathrm{E}$ lectron capture negative chemical ionization (ECNCI) mass spectrometry is an ionization technique that provides excellent sensitivity and considerable selectivity for the detection of organochlorine compounds in environmental matrices [1]. A common feature of the ECNCI spectrum of many of these chlorinated compounds is the presence of ions at $19 \mathrm{u}$ lower than the molecular ion [2-4]. These ions are formed in reactions between the organochlorine and trace amounts of oxygen present in the source and correspond to the addition of one oxygen atom with the simultaneous loss of one chlorine atom.

Many investigators have deliberately introduced oxygen into the source and have reported that the oxygen addition-induced dechlorination reactions could provide some structural information for polychlorinated diphenyl ethers [5], dibenzofurans [6], and dibenzodioxins [7]. Collision-induced dissociation

Address reprint requests to Dr. F. Lépine, Institut Armand-Frappier, 531 des Prairies Boulevard, Laval, Québec, Canada, H7N 4Z3.

- Presented in part at the 43rd ASMS Conference, Atlanta, GA, May 1995.
(CID) of the M-19 ions of a variety of polychlorinated biphenyls (PCB) congeners also was performed for the same purpose [8]. One objective of these experiments was to correlate the data with the chlorine substitution patterns of each congener, because the chromatographic separation of some of these congeners sometimes is difficult. Determination of the composition of a mixture of congeners is important in environmental studies, because toxicities vary enormously with their substitution pattern.

We observed that the dechlorination reactions of PCBs and various 1,1,1-trichloro-2,2-bis ( $p$-chlorophenyl)ethane (DDT) metabolites in a mass spectrometer were regioselective, especially under $\mathrm{ECNCI}$ conditions $[9,10]$, and it became of interest to see if such regioselectivity also could be observed for the oxygen addition-induced dechlorination of these compounds.

\section{Materials and Methods}

The simple syntheses of the various labeled DDT metabolites and PCBs were described previously $[10,11]$. For the PCBs, synthesis consisted of the chlo- 
rination, by using ${ }^{35} \mathrm{Cl}_{2}$, of the six symmetrical tetrachlorobiphenyls in an organic solvent. The resulting mixtures of compounds were analyzed by capillary gas chromatography-mass spectrometry (GC-MS) and the identity of each penta- and hexachlorobiphenyl was established by their retention times, by using the data of Mullin et al. [12], who published the retention times of all 209 PCBs. This also allowed us to determine the position of the ${ }^{35} \mathrm{Cl}$ labels within the molecules. The added label ${ }^{35} \mathrm{Cl}$ enrichment was $99.6 \%$, as determined by analysis of the molecular ion cluster in the electron impact mode [11]. Calculation of the percentage of residual label in dechlorinated ions of interest was performed by normalization of the intensities of $P+2$ and $P+4$ ions of a given cluster relative to the ${ }^{35} \mathrm{Cl}$ monoisotopic ion. This normalized profile then was compared with a computer-generated series of profiles that correspond to decreasing values of the ${ }^{37} \mathrm{Cl} /{ }^{35} \mathrm{Cl}$ ratio [11].

For the DDT metabolites, ${ }^{37} \mathrm{Cl}$-chlorobenzene first was synthesized and reacted with trichloroacetaldehyde to produce $p, p^{\prime}$ - and $o, p^{\prime}$-DDT. The resulting mixture was then gamma irradiated to produced $p, p^{\prime}$ and $0, p^{\prime}$-DDD, and treatment with base afforded $p, p^{\prime}$ DDE and $p, p^{\prime}$-DDMU. All these products were obtained as mixtures, but easily could be separated and identified by capillary GC-MS [10]. The added ${ }^{37} \mathrm{Cl}$ enrichment for the DDT metabolites was 94.6\% [10].

In this work, the various mixtures of ${ }^{35} \mathrm{Cl}$ - and ${ }^{37} \mathrm{Cl}$-labeled compounds were analyzed by GC-MS with a Hewlett-Packard (Avondale, PA) 5988A mass spectrometer by using a $30-\mathrm{m} \mathrm{DB}-1$ column. The PCB analyses were performed in triplicate. The reagent gas was a $20 \%$ mixture of oxygen in methane. Note that this mixture is explosive and that precautions must be taken to avoid sources of ignition. The purity of the methane used was greater than $99.99 \%$ (Linde, Montreal, Canada). The source pressure was 0.3 torr and the source temperature was $150{ }^{\circ} \mathrm{C}$. Because of an intense signal at $m / z 233-235$ due to oxidation of the Re filament $\left(\mathrm{ReO}_{3}\right)$, the scan range was $240-400 \mathrm{u}$. The DDT metabolites were analyzed under the same conditions.

\section{Results}

\section{PCBs}

To evaluate the regioselectivity of dechlorination, 6 penta- and 22 hexachloro-PCBs labeled at various positions with one or two ${ }^{35} \mathrm{Cl}$ were used. The regioselectivity of the dechlorination reaction is reflected by the residual amounts of label in the $\mathrm{M}-19$ ion cluster. In a previous experiment [9], methane alone was used as the moderator gas and the M-19 ions were observed, but their abundances were too small to allow reliable quantification of their labeled chlorine content. To increase the abundances of these ions, a $20 \%$ (by volume) oxygen in methane mixture was used in the present study. Under these conditions, the mass spectra of most of the PCBs consist almost exclusively of $\mathrm{M}-19$ ions.

Figure 1 presents the oxygen-methane ECNCI mass spectrum of $2,2^{\prime}, 3,3^{\prime}, 4^{35} \mathrm{Cl}, 4^{\prime}{ }^{35} \mathrm{Cl}$-hexachlorobiphenyl. This spectrum is one of the very few that show a molecular ion. The mass spectrum of the same compound, with methane as the moderator gas, is presented for comparison. The positions of the labels on the PCBs, the observed abundances of the $\mathrm{P}, \mathrm{P}+2$, and $P+4$ ions of the M-19 clusters, and the percentages of the label retained in the M-19 ions are presented in Table 1 . The $\mathrm{M}-19$ ions of all the pentachloro-PCBs retained between 77 and $79 \%$ of the label. For the hexachloro-PCBs, this value varied betwcen 81 and $84 \%$.

\section{DDT Metabolites}

Four DDT metabolites labeled with one ${ }^{37} \mathrm{Cl}$ on each aromatic ring were studied. These were 2,2-bis(4${ }^{37} \mathrm{Cl}$-chlorophenyl)-1-chloroethylene ( $p, p^{\prime}$-DDMU; Figure 2), 2,2-bis $\left(4-{ }^{37} \mathrm{Cl}\right.$-chlorophenyl)-1,1-dichloroethylene ( $p, p^{\prime}$-DDE; Figure 3), 2-(4- ${ }^{37} \mathrm{Cl}$-chlorophenyl)$2-\left(2-{ }^{37} \mathrm{Cl}\right.$-chlorophenyl)-1,1-dichloroethane ( $0, p^{\prime}$-DDD; Figure 4), and 2,2-bis $\left(4-{ }^{37} \mathrm{Cl}\right.$-chlorophenyl)-1,1-dichloroethane ( $p, p^{\prime}$-DDD; Figure 5). These compounds are known metabolites of commercial DDT preparations. As for the PCBs, because the M-19 ions of the DDT metabolites were not sufficiently intense to be ana-

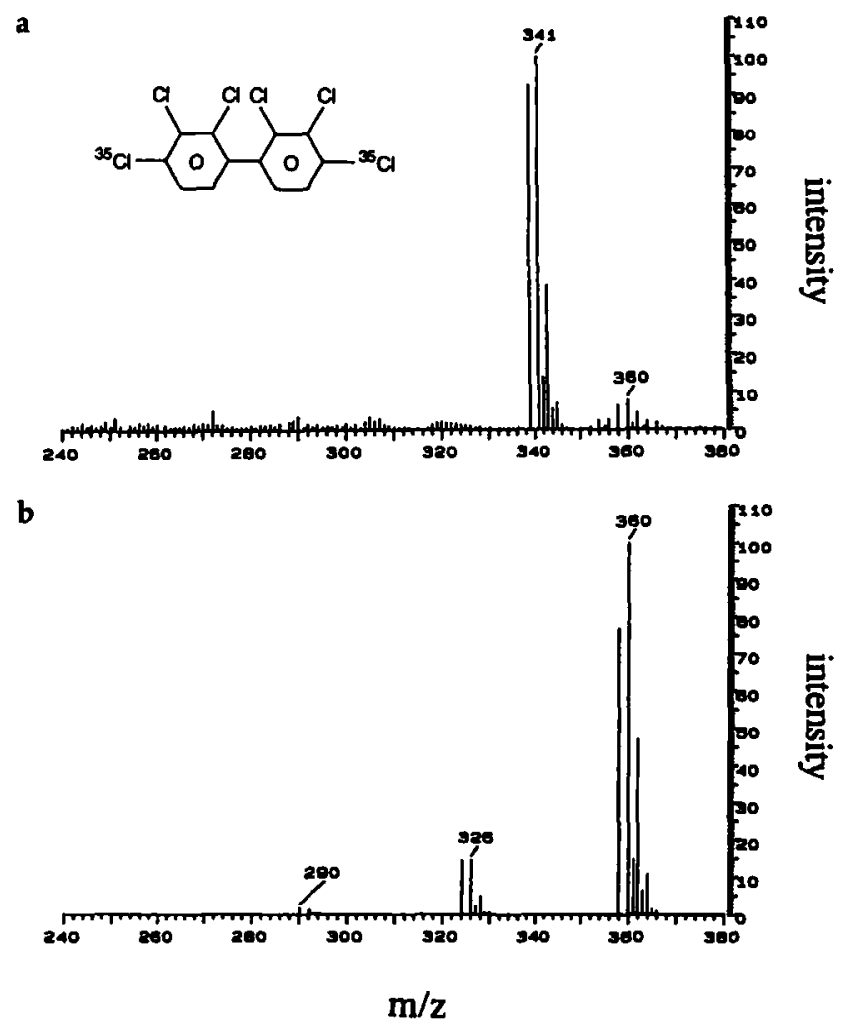

Figure 1. ECNCI spectra of $2,2^{\prime}, 3,3^{\prime}, 4^{35} \mathrm{Cl}, 4^{\prime}{ }^{35} \mathrm{Cl}$-hexachlorobiphenyl (IUPAC 128) with (a) oxygen-methane and (b) methane. 
Table 1. Percentage of residual label in the $\mathrm{M}-19$ ions of various ${ }^{35} \mathrm{Cl}$-labeled PCBs in ECNCI by using a $20 \%$ oxygen in methane mixture as reagent gas

\begin{tabular}{|c|c|c|c|}
\hline $\begin{array}{c}\text { IUPAC number } \\
\text { of unlabeled } \\
\text { PCBs }\end{array}$ & $\begin{array}{l}{ }^{35} \mathrm{Cl} \text { label position } \\
\text { in the labeled } \mathrm{PCB}\end{array}$ & $\begin{array}{l}\text { Abundances } \\
\text { of } P, P+2 \text {, } \\
\text { and } P+4 \\
\text { ions }^{a}\end{array}$ & $\begin{array}{c}\text { Percentage of } \\
\text { residual label } \\
\text { in } \mathrm{M}-\mathrm{Cl}+\mathrm{O} \\
\text { ions }\end{array}$ \\
\hline \multirow[t]{3}{*}{83} & $2,2^{\prime}, 3,3^{\prime}, 5^{35} \mathrm{Cl}$-penta- & 27,810 & 79 \\
\hline & & 29,018 & \\
\hline & & 10,669 & \\
\hline \multirow[t]{3}{*}{82} & $2,2^{\prime}, 3,3^{\prime}, 4^{35} \mathrm{Cl}$-penta- & 10,992 & 78 \\
\hline & & 11,992 & \\
\hline & & 5,011 & \\
\hline \multirow[t]{3}{*}{128} & $2,2^{\prime}, 3,3^{\prime}, 4^{35} \mathrm{Cl}, 4^{\prime 35} \mathrm{Cl}$-hexa- & 53,810 & 82 \\
\hline & & 60.893 & \\
\hline & & 24,405 & \\
\hline \multirow[t]{3}{*}{129} & $2,2^{\prime}, 3,3^{\prime}, 4^{35} \mathrm{Cl}, 5^{35} \mathrm{Cl}$-hexa- & 61,724 & 82 \\
\hline & & 68,801 & \\
\hline & & 28,190 & \\
\hline \multirow[t]{3}{*}{130} & $2,2^{\prime}, 3,3^{\prime}, 4^{35} \mathrm{Cl}, 5^{\prime}{ }^{35} \mathrm{Cl}$-hexa- & 86,387 & 82 \\
\hline & & 95,086 & \\
\hline & & 36,443 & \\
\hline \multirow[t]{3}{*}{131} & $2,2^{\prime}, 3,3^{\prime}, 4^{35} \mathrm{Cl}, 6^{35} \mathrm{Cl}$-hexa- & 267,829 & 83 \\
\hline & & 299,133 & \\
\hline & & 112,768 & \\
\hline \multirow[t]{3}{*}{132} & $2,2^{\prime}, 3,3^{\prime}, 4^{35} \mathrm{Cl}, 6^{\prime 35} \mathrm{Cl}$-hexa- & 129,049 & 83 \\
\hline & & 140,162 & \\
\hline & & 51,807 & \\
\hline \multirow[t]{3}{*}{133} & $2,2^{\prime}, 3,3^{\prime}, 5^{35} \mathrm{Cl}, 5^{\prime}{ }^{35} \mathrm{Cl}$-hexa- & 60,923 & 83 \\
\hline & & 63,507 & \\
\hline & & 23,543 & \\
\hline \multirow[t]{3}{*}{135} & $2,2^{\prime}, 3,3^{\prime}, 5^{35} \mathrm{Cl}, 6^{\prime}{ }^{35} \mathrm{Cl}$-hexa- & 228,605 & 83 \\
\hline & & 239,256 & \\
\hline & & 87,332 & \\
\hline \multirow[t]{3}{*}{136} & $2,2^{\prime}, 3,3^{\prime}, 6^{35} \mathrm{Cl}, 6^{\prime}{ }^{35} \mathrm{Cl}$-hexa- & 16,757 & 84 \\
\hline & & 16,737 & \\
\hline & & 6,058 & \\
\hline \multirow[t]{3}{*}{128} & $2,2^{\prime}, 3^{35} \mathrm{Cl}, 3^{\prime}{ }^{35} \mathrm{Cl}, 4,4^{\prime}$-hexa- & 4,495 & 84 \\
\hline & & 4,451 & \\
\hline & & 1,581 & \\
\hline \multirow[t]{3}{*}{138} & $2,2^{\prime}, 3^{35} \mathrm{Cl}, 4,4^{\prime}, 5^{\prime 35} \mathrm{Cl}$-hexa- & 14,045 & 84 \\
\hline & & 14,346 & \\
\hline & & 3,384 & \\
\hline \multirow[t]{3}{*}{153} & $2,2^{\prime}, 4,4^{\prime}, 5^{35} \mathrm{Cl}, 5^{\prime 35} \mathrm{Cl}$-hexa- & 15,015 & 84 \\
\hline & & 15,186 & \\
\hline & & 5,235 & \\
\hline \multirow[t]{3}{*}{136} & $2,2^{\prime}, 3^{35} \mathrm{Cl}, 3^{\prime}{ }^{35} \mathrm{Cl}, 6,6^{\prime}$-hexa- & 56,184 & 81 \\
\hline & & 65,056 & \\
\hline & & 27,883 & \\
\hline \multirow[t]{3}{*}{105} & $2^{35} \mathrm{Cl}, 3,3^{\prime}, 4,4^{\prime}$-penta- & 169,991 & 77 \\
\hline & & 198.969 & \\
\hline & & 80,796 & \\
\hline \multirow[t]{3}{*}{118} & $2^{35} \mathrm{Cl}, 3^{\prime}, 4,4^{\prime}, 5$-penta- & 336,262 & 78 \\
\hline & & 355,555 & \\
\hline & & 154,151 & \\
\hline \multirow[t]{3}{*}{128} & $2^{35} \mathrm{Cl}, 2^{\prime 35} \mathrm{Cl}, 3,3^{\prime}, 4,4^{\prime}$-hexa- & 4,966 & 83 \\
\hline & & 5,267 & \\
\hline & & 2,019 & \\
\hline
\end{tabular}


Table 1. (continued)

\begin{tabular}{|c|c|c|c|}
\hline $\begin{array}{l}\text { IUPAC number } \\
\text { of unlabeled } \\
\text { PCBs }\end{array}$ & $\begin{array}{l}{ }^{35} \mathrm{Cl} \text { label position } \\
\text { in the labeled } \mathrm{PCB}\end{array}$ & $\begin{array}{l}\text { Abundances } \\
\text { of } P, P+2 \text {, } \\
\text { and } P+4 \\
\text { ions }\end{array}$ & $\begin{array}{c}\text { Percentage of } \\
\text { residual label } \\
\text { in } \mathrm{M}-\mathrm{Cl}+\mathrm{O} \\
\text { ions }\end{array}$ \\
\hline \multirow[t]{3}{*}{153} & $2^{35} \mathrm{Cl}, 2^{\prime 35} \mathrm{Cl}, 4,4^{\prime}, 5,5^{\prime}$-hexa- & 18,934 & 83 \\
\hline & & 19,302 & \\
\hline & & 7,318 & \\
\hline \multirow[t]{3}{*}{167} & $2^{35} \mathrm{Cl}, 3^{\prime}, 4,4^{\prime}, 5,5^{\prime 35} \mathrm{Cl}$-hexa- & 16,341 & 82 \\
\hline & & 18,158 & \\
\hline & & 7,271 & \\
\hline \multirow[t]{3}{*}{138} & $2^{35} \mathrm{Cl}, 2^{\prime}{ }^{35} \mathrm{Cl}, 3,4,4^{\prime}, 5^{\prime}$-hexa- & 14,484 & 83 \\
\hline & & 15,282 & \\
\hline & & 5,677 & \\
\hline \multirow[t]{3}{*}{156} & $2^{35} \mathrm{Cl}, 3,3^{\prime}, 4,4^{\prime}, 5^{35} \mathrm{Cl}$-hexa- & 36,692 & 82 \\
\hline & & 42,066 & \\
\hline & & 17,183 & \\
\hline \multirow[t]{3}{*}{157} & $2^{35} \mathrm{Cl}, 3,3^{\prime}, 4,4^{\prime}, 5^{\prime 35} \mathrm{Cl}$-hexa- & 18,321 & 81 \\
\hline & & 21,243 & \\
\hline & & 9,024 & \\
\hline \multirow[t]{3}{*}{111} & $2{ }^{35} \mathrm{Cl}, 3,3^{\prime}, 5,5^{\prime}$-penta- & 144,538 & 78 \\
\hline & & 163,152 & \\
\hline & & 62,626 & \\
\hline \multirow[t]{3}{*}{127} & $3,3^{\prime}, 4^{35} \mathrm{Cl}, 5,5^{\prime}$-penta- & 22,582 & 77 \\
\hline & & 26,248 & \\
\hline & & 11,008 & \\
\hline \multirow[t]{3}{*}{159} & $2^{35} \mathrm{Cl}, 3,3^{\prime}, 4^{35} \mathrm{Cl}, 5,5^{\prime}$-hexa- & 23,184 & 81 \\
\hline & & 27,886 & \\
\hline & & 11,235 & \\
\hline \multirow[t]{3}{*}{162} & $2^{35} \mathrm{Cl}, 3,3^{\prime}, 4^{\prime}{ }^{35} \mathrm{Cl}, 5,5^{\prime}$-hexa- & 494,992 & 82 \\
\hline & & 553,625 & \\
\hline & & 219,965 & \\
\hline \multirow[t]{3}{*}{169} & $3,3^{\prime}, 4^{35} \mathrm{Cl}, 4^{\prime}{ }^{35} \mathrm{Cl}, 5,5^{\prime}$-hexa- & 49,070 & 82 \\
\hline & & 56,242 & \\
\hline & & 23,593 & \\
\hline \multirow[t]{3}{*}{133} & $2^{35} \mathrm{Cl}, 2^{\prime}{ }^{35} \mathrm{Cl}, 3,3^{\prime}, 5,5^{\prime}$-hexa- & 639,255 & 84 \\
\hline & & 654,801 & \\
\hline & & 221,851 & \\
\hline
\end{tabular}

a These values represent the averaged abundances of the ions of the cluster that corresponds to $M-19$. $P$ is the ion in which all the chlorines are ${ }^{35} \mathrm{Cl}$. The abundances of $P, P+2$, and $P+4$ are used to determine the amount of residual label in the $\mathrm{M}-19$ ions [10].

lyzed with methane, the same $20 \%$ oxygen in methane mixture was used. However, under these conditions, extensive fragmentation of the higher mass ions was evident and led to low intensities for the ions of interest, which did not allow accurate measurement of the residual label in these ions. However, qualitative information could be deduced from these spectra. The structure and mass spectra of the labeled DDT metabolites are presented in Figures 2-5. The spectra of the unlabeled compounds, taken under the same conditions, also are presented for comparison. The profiles of the major ion clusters of these spectra show that, in every case, the aromatic chlorines are retained; because of this, and to simplify the description of these ion clusters, they will be referred to by the most abundant ion of the cluster.
The spectrum of labeled $p, p^{\prime}$-DDMU $\left(\mathrm{M}^{--}=m / z\right.$ 286; not visible in Figure 2) includes ions at $m / z 282$ $(\mathrm{M}-4), 267$ (M-19), and 254. No molecular ion or fragments that correspond to the loss of one $\mathrm{Cl}$, as obtained with methane [10], were observed. The $\mathrm{M}-4$ ion (Figure 6,5) is probably produced by the addition of $\mathrm{O}_{2}$ with loss of $\mathrm{HCl}$. Structure $4 \mathrm{a}$ and its resonance form $4 b$ (Figure 6; $R=H$ ) are proposed for the $m / z$ 267 ion and take into account that the formation of this ion is by the loss of an aliphatic chlorine. The intense ion at $m / z 254$ probably is related structurally to $4,4^{\prime}$-dichlorobenzophenone 6 , and could be formed from the $m / z 282$ ion 5 through loss of $\mathrm{CO}$ or by cycloaddition of $\mathrm{O}_{2}^{--}$to the molecule, which forms the four-membered ring intermediate $3(R=H)$ that eliminates formyl chloride. 
a

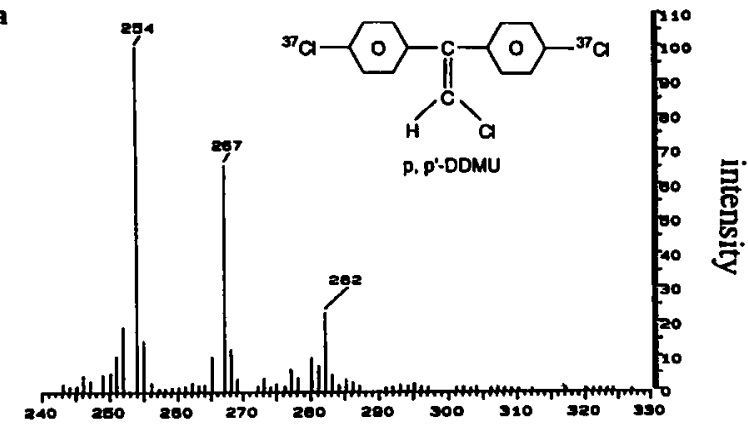

b

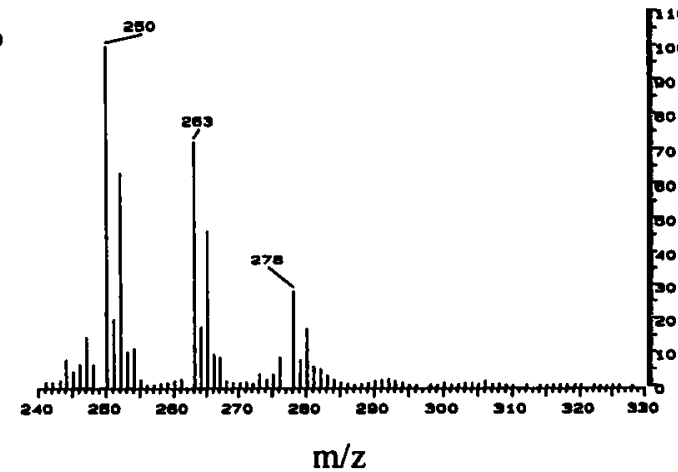

Figure 2. Oxygen-methane ECNCI spectra of (a) labeled $p, p^{\prime}-$ DDMU and (b) unlabeled $p, p^{\prime}$-DDMU.

The spectrum of the labeled $p, p^{\prime}-\mathrm{DDE}\left(\mathrm{M}^{--}=m / z\right.$ 320; Figure 3) includes ions at $m / z 301$ (M-19), 285 (M-35), 273 (M-47), and 254. The molecular ion of 1 (Figure 6) and the M-35 ion 2 also were observed in the spectrum obtained with methane [10]. The structures

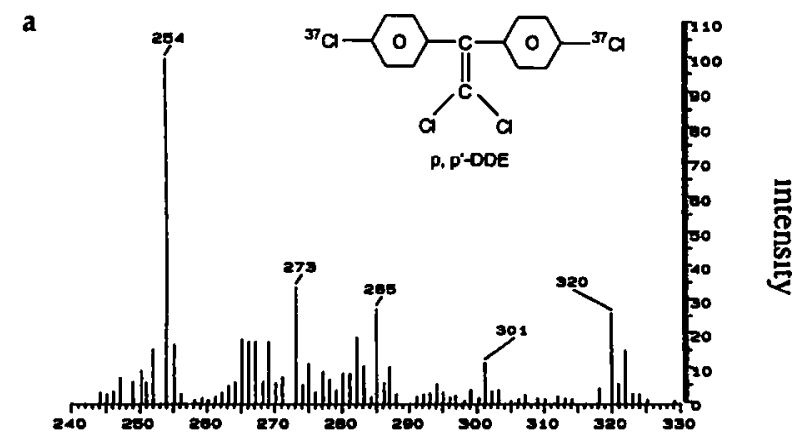

b

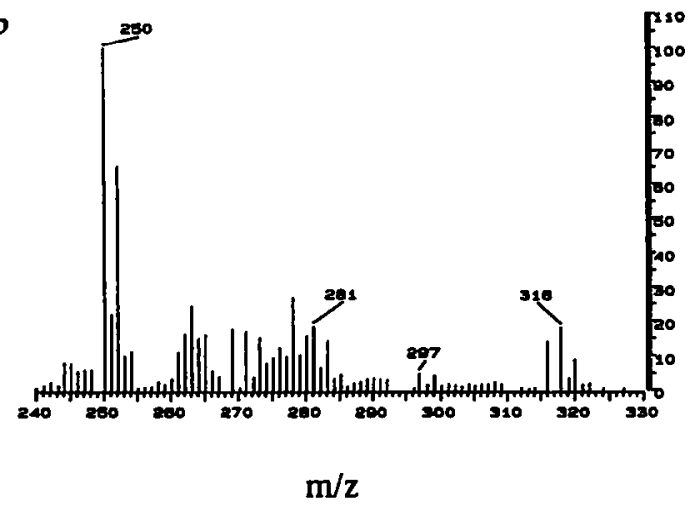

Figure 3. Oxygen-methane ECNCI spectra of (a) labeled $p, p^{\prime}$ DDE and (b) unlabeled $p, p^{\prime}$-DDE.
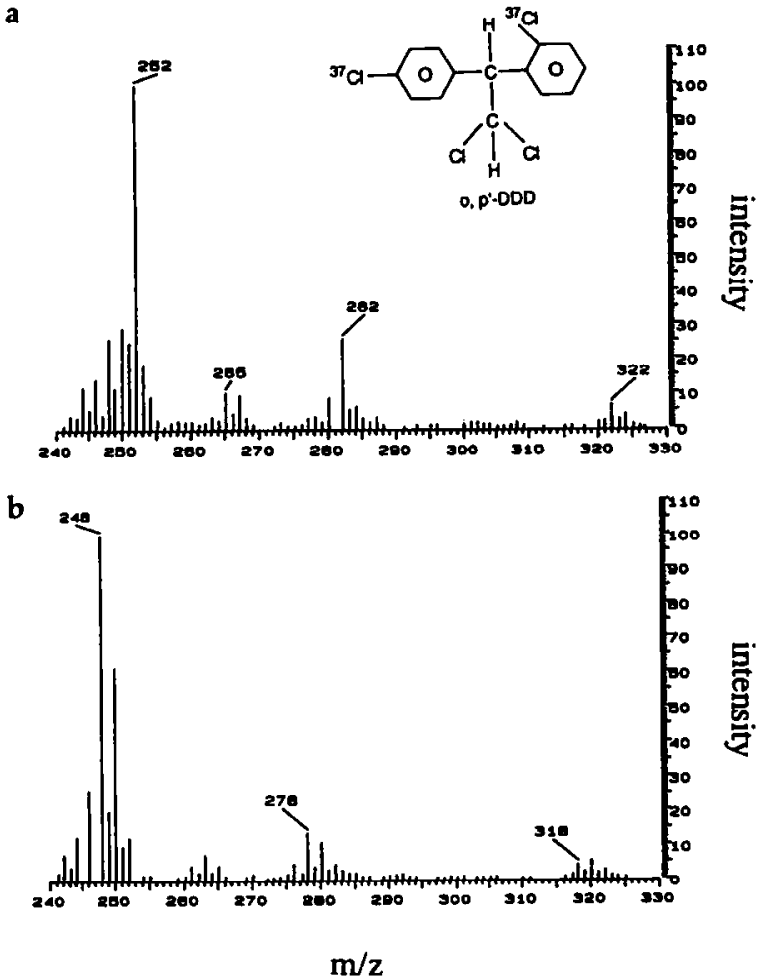

Figure 4. Oxygen-methane ECNCI spectra of (a) labeled $o, p^{\prime}-$ $\mathrm{DDD}$ and (b) unlabeled $o, p^{\prime}$-DDD.

4a and $\mathbf{4 b}$ are proposed for the $m / z 301$ ion, by analogy with the $m / z 267$ ion of $p, p^{\prime}$-DDMU. The $m / z 301$ ion $\mathbf{4 a}, \mathbf{b}$ is probably the precursor of $\mathbf{7}(\mathrm{m} / \mathrm{z}$ 273) through loss of $\mathrm{CO}$ (Figure 6; $\mathrm{R}=\mathrm{Cl}$ ). Formation of 6 in the $p, p^{\prime}$-DDE spectrum could be explained by
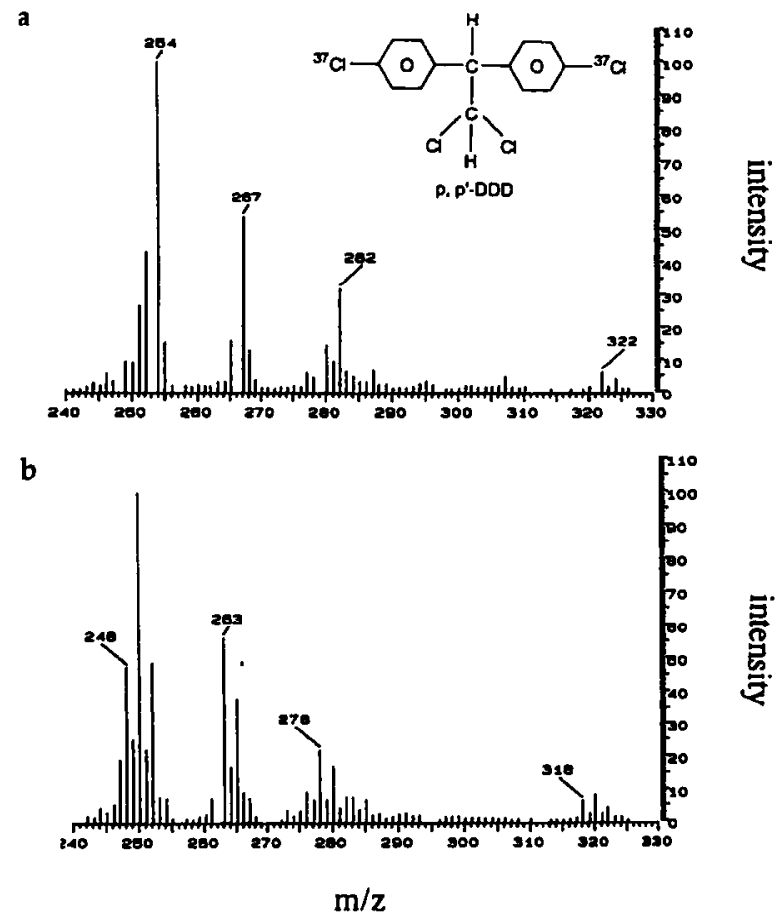

Figure 5. Oxygen-methane ECNCI spectra of (a) labeled $p, p^{\prime}$ DDD and (b) unlabeled $p, p^{\prime}$-DDD. 

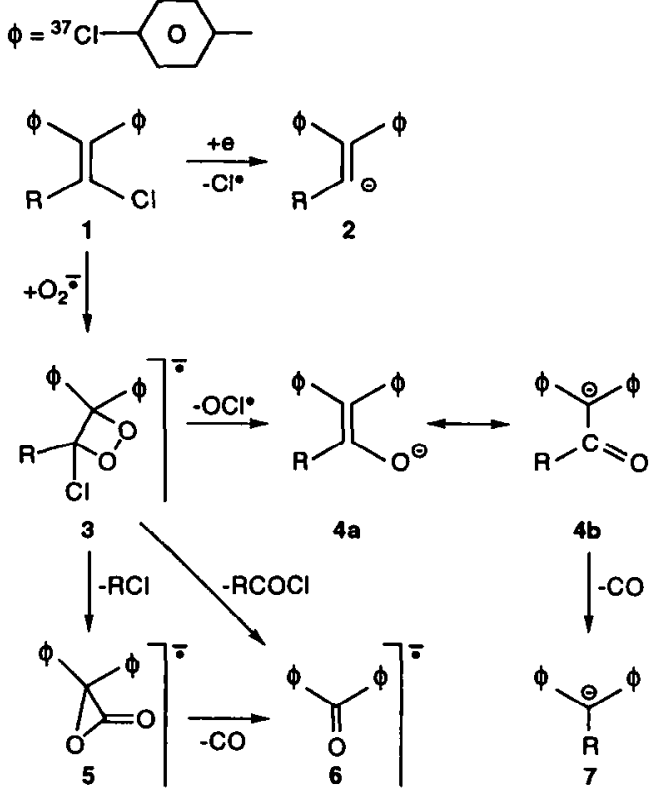

\begin{tabular}{lcccccccc} 
& & 1 & 2 & 3 & $4 a, b$ & 5 & 6 & 7 \\
\hline p,p'-DDMU & $R=H$ & $286^{b}$ & $251^{b}$ & $318^{a}$ & 267 & 282 & 254 & \\
p,p'-DDE & $R=C l$ & 320 & 285 & $352^{a}$ & 301 & 282 & 254 & 273
\end{tabular}

- lons presumed but not apparent

b Not observed

Figure 6. Proposed decomposition pathways of $p, p^{\prime}$-DDMU and $p, p^{\prime}-\mathrm{DDE}$.

the formation of 3 with the subsequent loss of phosgene $(\mathrm{R}=\mathrm{Cl})$.

The spectrum of labeled $0, p^{\prime}-\mathrm{DDD}\left(\mathrm{M}^{\cdot-}=m / z\right.$ 322; Figure 4) shows molecular $m / z 282(\mathrm{M}-40)$ and 252 (M-70) ions. The $m / z 282$ ion corresponds to addition of $\mathrm{O}_{2}$ to the molecular ion with the loss of two $\mathrm{HCl}$ molecules. This is equivalent to loss of a second molecule of $\mathrm{HCl}$ from the $\mathrm{M}-4$ ion in the $p, p^{\prime}$-DDMU spectrum. The molecular and the $m / z 252$ ions also were observed when methane was used [10]. In the present experiment, the $\mathrm{m} / \mathrm{z} 252$ ion cluster contains almost exclusively two ${ }^{37} \mathrm{Cl}$, which indicates that the two chlorines that are lost are aliphatic. The same phenomenon was observed for this ion when methane was used. The $m / z 254$ ion, which was observed for all of the other DDT metabolites, was not present in this spectrum.

The spectrum of the labeled $p, p^{\prime}$-DDD (Figure 5) includes ions at $m / z 282,267$, and 254 and is almost identical to the $p, p^{\prime}$-DDMU spectrum. It is likely that in a first step, $p, p^{\prime}$-DDD undergoes loss of $\mathrm{HCl}$ to produce a molecular ion of $p, p$-DDMU. Because the spectra of these two compounds are completely different when methane is used as the moderator gas [10], the initial loss of $\mathrm{HCl}$ observed here probably arises from abstraction of the relatively acidic $\alpha$ hydrogen, possibly by a superoxide $\mathrm{O}_{2}^{--}$radical anion or related species, followed by loss of chlorine. The degradation pathways of $o, p^{\prime}$ - and $p, p^{\prime}$-DDD are then quite different although dechlorination proceeds only at the aliphatic chlorines in both cases.

\section{Discussion}

\section{PCBs}

In a previous publication, the dechlorination reaction of PCBs in ECNCI was reported to be highly regioselective using methane as the moderator gas [9]. Under these conditions, the labeled PCBs all presented abundant molecular and M-34 ions, which indicated that the addition of hydrogen was involved in the course of the dechlorination reaction. In the present experiment, under similar conditions but for the presence of oxygen in the source, the results presented in Table 1 indicate that the oxygen addition-induced dechlorination is completely random. Label retentions are very close to 80 and $83 \%$ for the penta- and hexachloro congeners, respectively, and correspond to random loss of one chlorine atom out of the five or six initially present. This observation also is confirmed by the residual label content for PCB congeners that have the same chlorine substitution pattern but different label positions, as in congeners $128,138,153$, and 133 . As for the ECNCI experiment that uses methane, no isotope effect could be detected.

Two mechanisms were postulated to explain the formation of the M-19 ions observed in the ECNCI spectra of a variety of chlorinated compounds [13]. The first mechanism involves the radical anion of the compound that reacts with $\mathrm{O}_{2}$ to produce the $\mathrm{MO}_{2}$ radical anion (eq 1), which then undergoes loss of a $\mathrm{ClO}$ radical that produces the $\mathrm{M}-19$ ions. In such a mechanism, the lack of regioselectivity observed in the present study with PCBs could be accounted for by delocalization of the captured electron on the biphenyl system and reaction with $\mathrm{O}_{2}$ followed by random loss of $\mathrm{Cl}$ as $\mathrm{ClO}^{\circ}$ :

$$
\begin{aligned}
& \mathrm{M}^{--}+\mathrm{O}_{2} \rightleftarrows \mathrm{MO}_{2}^{--} \\
& \mathrm{M}+\mathrm{O}_{2}^{--} \rightleftarrows \mathrm{MO}_{2}^{--} \\
& \mathrm{M}+\mathrm{O}_{2}^{--} \rightleftarrows \mathrm{M}^{--}+\mathrm{O}_{2}
\end{aligned}
$$

The second mechanism involves the reaction of superoxide $\left(\mathrm{O}_{2}^{-}\right)$with the neutral PCB molecule to produce the $\mathrm{MO}_{2}$ radical anion (eq 2). Knighton et al. [14], who used an oxygen-methane mixture as the reagent gas, demonstrated that this mechanism was not responsible for the formation of the M-19 ions for a variety of chlorinated compounds. However, the oxygen concentrations they used (less than $2000 \mathrm{ppm}$ ) were much lower than in the present work $(20 \%)$. At higher oxygen concentrations, the probability of electron capture by oxygen increases, and this increase could increase the proportion of the M-19 ions formed by attack by superoxide on the neutral PCBs. 
Charge exchange between superoxide and the neutral compound (eq 3) also was invoked as another reason why the second mechanism was not important in Knighton's study. The extent of charge exchange is controled, in part, by the electron affinities of the two reactants [15]. For oxygen, this value was found to be $0.44 \mathrm{eV}$ [16]. There are no data available on the electron affinities of PCBs, but Knighton reported that the electron affinities of hexachlorobenzene and pentachlorobenzene were 1.0 and $0.7 \mathrm{eV}$, respectively [14]. Because a decrease in the number of chlorines on the aromatic ring decreases the electron affinity, and assuming one can extrapolate the results obtained with a simple aromatic ring to a biphenyl system, one can estimate that the electron affinities of the PCBs listed in Table 1, which contain an average of three chlorine atoms on each aromatic ring, are not much larger than that of oxygen. Because of the relatively large amount of oxygen in the source, charge exchange is probably not sufficient to decrease the concentration of superoxide to a point that it should not be considered an important reactant in the formation of the M-19 ions. The absence of molecular ions in the spectra of most PCBs also favors eq 2, and the observed lack of regioselectivity could be due to random attack by superoxide at any chlorine-bearing biphenyl carbon. It is also possible that under these reaction conditions, both mechanisms are important.

\section{DDT Metabolites}

In contrast to the PCBs, all the dechlorination reactions observed with the DDT metabolites were completely regiospecific: only the aliphatic chlorines were lost. The regioselectivity of these reactions is probably related to the ability of the diphenylmethane moiety to stabilize a radical and/or a negative charge by resonance through the two aromatic rings. Only $p, p^{\prime}$ DDMU and $p, p^{\prime}$-DDE presented M-19 ions. For these two ions, oxygen addition and dechlorination occur at the carbon beta to the aromatic rings, which allows delocalization of the charge on the aromatic rings through the resonance structure $4 b$ (Figure 6 ). The capacity of an ion that is structurally related to $4,4^{\prime}$ dichlorobenzophenone 6 to stabilize a negative charge probably also accounts for the abundance of the $m / z$ 254 ion in the spectra of the alpha-beta unsaturated DDT metabolites.

$4,4^{\prime}$-Dichlorobenzophenone also is produced in the decomposition of many DDT metabolites by gamma irradiation in aliphatic solvents in the presence of oxygen [17]. Gamma irradiation generates solvated electrons in the solvent. In the presence of oxygen, the superoxide radical is generated and probably adds at the alpha carbon to ultimately produce 4,4-dichlorobenzophenone. The results obtained in the present experiment demonstrate that there is a close parallel between these solvent and gas-phase reactions.

\section{Conclusion}

The regioselectivity of dechlorination reactions of various organochlorines in ECNCI in the presence of oxygen is very dependent upon the structure of the organochlorine. Contrary to what was observed previously with methane alone as the moderator gas, PCBs were dechlorinated randomly in the presence of oxygen. DDT metabolites, on the other hand, were dechlorinated regioselectively at the aliphatic sites.

\section{Acknowledgments}

This work was supported by grants from the Natural Sciences and Engineering Research Council of Canada and from the Medical Research Council of Canada.

\section{References}

1. Ong, V. S.; Hites, R. A. Mass Spectrom. Rev. 1995, 13, 259.

2. Biros, F. J.; Dougherty, R. C.; Dalton, J. Org. Mass Spectrom. 1971, 6, 1161.

3. Levonowich, P. F.; Tannenbaum, H. P.; Dougherty, R. C. J. Chem. Soc. Chem. Commun. 1975, 597.

4. Stemmler, E. A.; Hites, R. A.; Arbogast, B.; Budde, W. L.; Deinzer, M. L.; Dougherty, R. C.; Eichelberger, J. W.; Foltz, R. L.; Grimm, C.; Grimsrud, E. P.; Sakashita, C.; Sears, L. J. Anal. Chem. 1988, 60, 781.

5. Busch, K. L.; Norstrom, A.; Bursey, M. M.; Hass, J. R.; Nilsson, C.-A. Biomed. Mass Spectrom. 1979, 6, 157.

6. Fung, D.; Boyd, R. K.; Safe, S.; Chittim, B. G. Biomed. Mass Spectront. 1985, 12, 247.

7. Hass, R. J.; Friesen, M.; Hoffman, M. K. Org. Mass Spectrom. $1979,14,9$.

8. Guevremont, R.; Yost, R. A.; Jamieson, W. D. Biomed. Environ. Mass Spectrom. 1987, 14, 435.

9. Lépine, F. L.; Milot, S.; Reimer, M. L. J.; Mamer, O. A. Org. Mass Spectrom. 1994, 29, 133.

10. Lépine, F. L.; Milot, S.; Mamer, O. A. J. Mass Spectrom. 1995, 30, 885.

11. Lépine, F. L.; Milot, S.; Reimer, M. L. J.; Mamer, O. A. Org. Mass Spectrom. 1992, 27, 1311.

12. Mullin, M. D.; Pochini, C. M.; McCrindle, S.; Romkes, M.; Safe, S. H.; Safe, S. Environ. Sci. Technol. 1984, 205, 103.

13. Dzidic, I.; Carroll, D. I.; Stillwell, R. N.; Horning, E. C. Anal. Chem. 1975, 47, 1308.

14. Knighton, W. B.; Bognar, J. A.; Grimsrud, F. P. I. Mass Spectrom. 1995, 30, 557.

15. Grimsrud, E. P.; Chowdhury, S.; Kebarle, P. Int. J. Mass Spectrom. Ion Processes 1986, 68, 57.

16. Janousek, B. K.; Brauman, J. I. Gas Phase Ion Chemistry, Vol. 2; Bowers, M., Ed.; Academic Press: New York, 1979; p 53.

17. Lépine, F. L.; Brochu, F.; Milot, S.; Mamer, O. A.; Pépin, Y. J. Agric. Food Chem. 1994, 42, 2012. 